\title{
Unsteady characteristics of cloud cavitating flow near the free surface around an axisymmetric projectile
}

\author{
Yiwei Wang, Xiaocui Wu, Chenguang Huang*, Xianqian Wu \\ Key Laboratory for Mechanics in Fluid Solid Coupling Systems, Institute of Mechanics, Chinese Academy of Sciences, Beijing 100190, China
}

\section{A R T I C L E I N F O}

\section{Article history:}

Received 10 October 2015

Revised 21 April 2016

Accepted 21 May 2016

Available online 26 May 2016

\section{Keywords:}

Cloud cavitating flow

Free surface

Large eddy simulation

Cavity shedding

Wave elevation

\begin{abstract}
A B S T R A C $T$
The effect of free surface on unsteady cloud cavitation is important for high-speed surface vehicles, However, previously published experimental and numerical works regarding this topic are limited. In this paper, a typical launching experiment is performed with the presence of free surface. A numerical approach is established by using large eddy simulation and volume-of-fluid methods. Firstly, unsteady evolutions of the cavity and re-entry jet are obtained in both experimental and numerical results, which agree well with each other. Results indicate that the cavity evolution on the upper side of projectile is remarkably different compared to the lower side under the free-surface effect. For instance, on the upper side, cavity growth is slower, the velocity of the re-entry jet is higher, the cavity sheds faster, and the position of shedding cavity collapse is closer to the main cavity. Secondly, the effect of the free surface is studied by analyzing the constraint variation. Because the flow stream around the upper surface is thin, changing its direction under the effect of pressure difference inside and outside the cavity is easy. Non-axisymmetric collapse features generate a mass of strong vortexes on the cylindrical surface, and the non-uniform distribution of high pressure region is also one of the most important factors to induce lateral and vertical forces on the projectile. Finally, the heights of wave elevation in cases with and without cavitation are compared. The presence of cavitation leads to an increase in wave height, but the increment is about half the thickness of the cavity. This finding indicates that the actual constraint effect is between the effects of the infinite water field and the fully free condition.
\end{abstract}

(c) 2016 Elsevier Ltd. All rights reserved.

\section{Introduction}

Cavitation is one of the main obstacles in increasing the speed of surface vehicles. In particular, when the interaction between free surface and cloud cavitating flow is involved, the problem becomes complicated. On the one hand, the free surface may affect the dynamic behavior of cloud cavitation such as development and shedding. On the other hand, the evolution of cloud cavitation region can affect wave elevation as well. Relevant studies in literature are limited and the understanding of the interactions is still inadequate (Faltinsen, 2005).

By contrast, a considerable amount of research has been conducted on cloud cavitating flow by neglecting the effect of free surface. Experimental research is mostly conducted in cavitation water tunnels.The flow motion and structure such as cavity shedding

\footnotetext{
* Corresponding author.

E-mail address: huangcg@imech.ac.cn (C. Huang).
}

and re-entry jet are investigated by using the high-speed camera, particle image velocimetry and X-ray (Stutz and Legoupil, 2003; Stutz and Reboud, 1997, 2000). These tools are important in revealing mechanisms and validating the computational results. Several cavitation models used by numerical simulations have been established in the framework of homogeneous multiphase flow to describe the mass transfer of phase change, and many applications have been achieved (Merkle et al., 1998; Singhal et al., 2002; Kunz et al., 1998). In simulations of turbulence effect, Reynolds averaged Navier-Stokes equations and turbulence models with physical modifications have been widely used for engineering applications. Approaches such as modified renormalization-group $k-\varepsilon$ turbulence model (Coutier-Delgosha et al., 2007a, 2003a, 2003b, 2007b; Zhou and Wang, 2008), filter-based model (FBM) (Wu et al., 2005), partially averaged Navier-Stokes (PANS) method ( et al., 2014; Huang and Wang, 2011; Ji et al., 2014, 2013), are widely used. In recent years, large eddy simulation (LES), which can capture considerable details of large-scale turbulent eddies in the flow field with high accuracy, has been adopted for research on cavitating flows and some promising results have been published 
(Bensow and Bark, 2010; Dittakavi et al., 2010; Gnanaskandan and Mahesh, 2015; Huang et al., 2014; ji et al., 2015; Roohi et al., 2013; Wang and Ostoja-Starzewski, 2007; Yu et al., 2014).

Research on the effect of free surface on cavitation is lacking in literature. Producing a stable free surface is difficult in a cavitation water tunnel, which is why credible experimental results are rare. There were some early experiments carried out by Dawson (1959). They studied the evolution of supercavitating flow around a wedge-shape hydrofoil near the free surface and obtained the overall forces as one of the results. However, the cavitating flow in the experiments was generated by ventilation under low-velocity restriction in the tunnel. Theoretical analysis and numerical simulation are the primary research methods used at present. Potential flow theory and volume-of-fraction (VOF) method are often adopted to study the effect of free surface on the flow field around hydrofoils (Karim et al., 2014; Liang et al., 2013; Xie and Vassalos, 2007). Early research which investigates the effect of submersion depth on the length of supercavitation based on linearized theory can also be found in the book of Franc and Michel (2005). Based on consistency between theoretical models on the free surface and cavitation, Faltinsen and Semenov (2008) established a numerical approach within a unified framework on the supercavitating flow near the free surface. The effects of depth, Froude number, cavitation number and other parameters of cavitation shape and lift based on calculation results are discussed. Bal et al. presented a boundary element method (Bal, 2007, 2011; Bal and Kinnas, 2002) for cavitating hydrofoils near a free surface, which was also extended to the applications of surface piercing cavitating hydrofoils, including a tandem case. The effects of Froude number, cavitation number and submergence depth of the hydrofoil from free surface have also been discussed. The effect of the free surface was also considered in some research on supercavitating flow in shallow water (Amromin, 2007; Chen et al., 2011). The aforementioned works are mostly limited to the study of stable cavitation, particularly supercavitation. However, research on instable cavitation near the free surface is still very difficult, because appropriate means of validation are inadequate.

In this paper, a typical launching experiment is performed, and a numerical approach using VOF and LES methods on the cloud cavitating flow near the free surface is established. Experiments and simulations are performed on typical cases around an axisymmetric projectile. Numerical methods are validated by comparing results with underwater launching experiments. The unsteady nonaxisymmetrical characteristics of cavity evolution are obtained. The effects of free surface on re-entry jets, cavity shedding and the effects of cavities on wave elevation are both studied.

\section{Experimental setup}

\subsection{System principle}

The launching system is established on the basis of the SHPB technology (Wei et al., 2011) . Projectiles are driven by the stress wave by using the SHPB system (as shown in Fig. 1). The projectile can be transiently accelerated to reach $30 \mathrm{~m} / \mathrm{s}$ in less than $50 \mu \mathrm{s}$. It is very difficult to generate a free surface and keep it undisturbed before flowing around the model in a cavitation water tunnel. However, by using the present method, the free surface keeps static before launching. The distance that the projectile moves during the acceleration process is less than $3 \%$ of the projectile diameter. Therefore the disturbance during launching is very slight and can be neglected. It is appropriate for investigating the cavitating flow near the free surface.

The present experimental method still has some disadvantages. The launching speed of model is set by adjusting the pressure in the air chamber with high pressure. However, the propagation

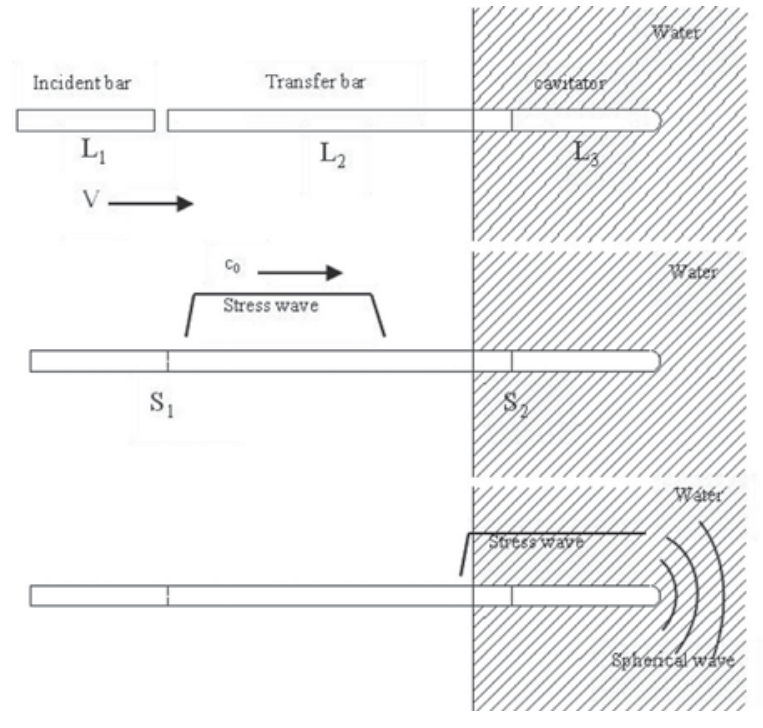

Fig. 1. Launch process schematic

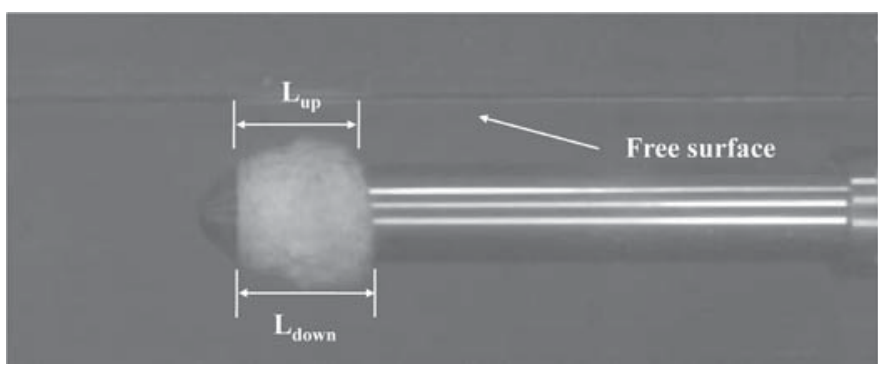

Fig. 2. Typical cavitation photograph.

of stress wave is insensitive to contact and friction conditions, so the launching speed disperses under the same pressure in the air chamber. Actually, exact speed values need to be obtained by analyzing the model motion in images.

\subsection{Projectile model and typical experimental condition}

The projectile used in this study is a slender cylinder with a conical head and made of polished stainless steel. The total length is $246 \mathrm{~mm}$, the diameter is $37 \mathrm{~mm}$ and the conical angle is $90^{\circ}$.

As shown in Fig. 2, a photograph of typical cavitation can be obtained using a high-speed camera with 25,000 frames per second(FPS). In a typical experiment, the distance between the free surface and the upper side of the projectile is $17 \mathrm{~mm}$, and an analysis of obtained images indicates that the speed is approximately uniform at $17.8 \mathrm{~m} / \mathrm{s}$. The temperature is $20^{\circ} \mathrm{C}$. The cavitation number can be calculated as

$\sigma=\frac{p_{\infty}-p_{v}}{\frac{1}{2} \rho_{l} v_{\infty}^{2}}=0.62$

where $p_{\infty}$ is the pressure in open air, $p_{v}$ is the saturated vapour pressure,$\rho_{l}$ is the liquid water density and $v_{\infty}$ is the speed of projectile. Under the present condition, the projectile is small and fast, the pressure difference between the upper and lower regions caused by gravity is much smaller than the flow dynamic pressure, as $\frac{\rho_{g} g d}{\frac{1}{2} \rho_{l} v_{\infty}^{2}}=0.0023 \ll 1$. Therefore, the variation in cavitation number in the $y$ direction caused by gravity can be neglected.

The shoulder and tail cavities with shedding bubbles are clearly shown. The cavity is nonaxisymmetrically affected by the free surface. Therefore, the length on the upper and lower sides of the cavity is measured, as shown in Fig. 2. The precision of the length and 


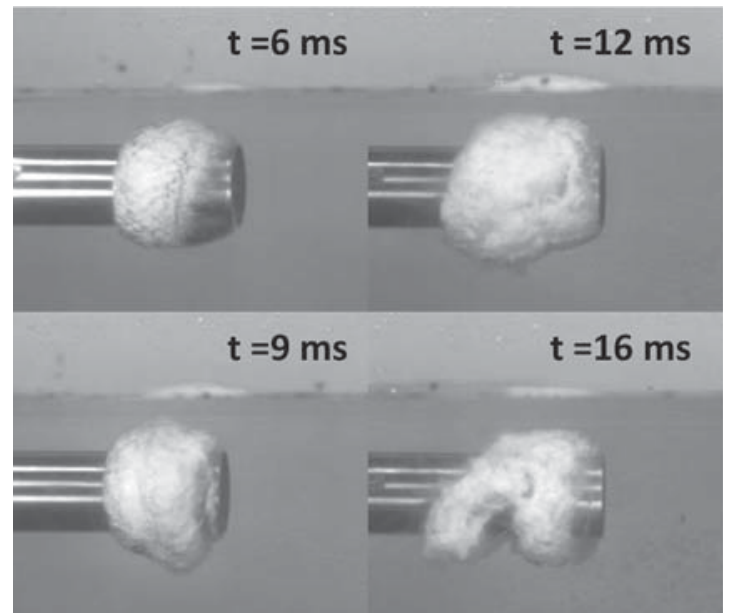

Fig. 3. Evolutions of the cavity and free surface at typical moments around a blunt body. Developments of the cavity and re-entry jet are at the moment of 6 ms. The re-entry jet arrives at the leading edge of the projectile at the moment of $9 \mathrm{~ms}$. Cavity Shedding is at the moment of $12 \mathrm{~ms}$. Collapse of the shedding cavity is at the moment of $16 \mathrm{~ms}$.

thickness is approximately a pixel of the image, which stands for $0.74 \mathrm{~mm}$. In the following sections, the evolution of shoulder cavities will be mainly discussed based on the experimental pictures and numerical results.

In order to verify the repeatability of the present experiment, other experiments with similarly shaped models and similar speed are also performed, and these typical phenomena also appear. For example, sheet and cloud cavities in the flow around a blunt body with the speed of $18 \mathrm{~m} / \mathrm{s}$ are shown in Fig. 3. The moments are delayed compared to the flow around the conical projectile because the cavity is longer in this case.

\section{Numerical methods}

\subsection{Governing equations}

To simulate the motions of liquid water, vapor and air, mixture/multiphase flow equations are adopted. Continuity and momentum equations for the mixture are established as follows:

$$
\begin{aligned}
& \frac{\partial \rho}{\partial t}+\frac{\partial\left(\rho u_{j}\right)}{\partial x_{j}}=0 \\
& \frac{\partial\left(\rho u_{i}\right)}{\partial t}+\frac{\partial\left(\rho u_{i} u_{j}\right)}{\partial x_{j}}=-\frac{\partial p}{\partial x_{j}}+\frac{\partial}{\partial x_{j}}\left(\mu \frac{\partial u_{i}}{\partial x_{j}}\right)
\end{aligned}
$$

where $u_{i}$ is the velocity component in the $i$ direction, $\rho$ is the mixture density, and $p$ is the pressure. Laminar viscosity $\mu$ is defined as a volume-weighted average of the three components as

$\mu=\left(1-\alpha_{v}-\alpha_{a}\right) \mu_{l}+\alpha_{v} \mu_{v}+\alpha_{a} \mu_{a}$

where $\alpha$ is the volume fraction of each phase. Subscripts $l, v, a$ represent phases of liquid, vapor and air, respectively. Mixture density $\rho$ is defined as

$\rho=\left(1-\alpha_{v}-\alpha_{a}\right) \rho_{l}+\alpha_{v} \rho_{v}+\alpha_{a} \rho_{a}$.

The volume fractions of vapor and air are governed by the following transport equations:

$$
\begin{aligned}
& \frac{\partial\left(\rho_{v} \alpha_{v}\right)}{\partial t}+\frac{\partial\left(\rho_{v} \alpha_{v} u_{j}\right)}{\partial x_{j}}=\dot{m}^{+}-\dot{m}^{-} \\
& \frac{\partial\left(\rho_{a} \alpha_{a}\right)}{\partial t}+\frac{\partial\left(\rho_{a} \alpha_{a} u_{j}\right)}{\partial x_{j}}=0
\end{aligned}
$$

In Eqs. (6), $\dot{m}^{+}$and $\dot{m}^{-}$represent the mass transfer rate of evaporation and condensation, which are derived from the bubble dynamics equations of generalized Rayleigh-Plesset equation by Zwart et al. (2004) as follows.

$$
\begin{aligned}
& \dot{m}^{+}=F_{v a p} \frac{3 a_{n u c}\left(1-\alpha_{v}\right) \rho_{v}}{R_{B}} \sqrt{\frac{2}{3} \frac{\max \left(p_{v}-p, 0\right)}{\rho_{l}}} \\
& \dot{m}^{-}=F_{\text {cond }} \frac{3 \alpha_{v} \rho_{v}}{R_{B}} \sqrt{\frac{2}{3} \frac{\max \left(p-p_{v}, 0\right)}{\rho_{l}}}
\end{aligned}
$$

In Eqs. (8) and (9) generalized bubble radius $R_{B}$ is set at $10^{-6} \mathrm{~m}$, the saturated vapor pressure $p_{v}$ is set as $2340 \mathrm{~Pa}$, nucleation site volume fraction $a_{n u c}$ is set as $5 \times 10^{-4}$, the evaporation coefficient $F_{\text {vap }}$ is set as 50 and the condensation coefficient $F_{\text {cond }}$ is set as 0.01 .

\subsection{LES approach}

Applying a Favre-filtering operation to Eqs. (2) and (3) derives the following LES equations:

$$
\begin{aligned}
& \frac{\partial \rho}{\partial t}+\frac{\partial\left(\rho \bar{u}_{j}\right)}{\partial x_{j}}=0 \\
& \frac{\partial\left(\rho \bar{u}_{i}\right)}{\partial t}+\frac{\partial\left(\rho \bar{u}_{i} \bar{u}_{j}\right)}{\partial x_{j}}=-\frac{\partial \bar{p}}{\partial x_{j}}+\frac{\partial}{\partial x_{j}}\left(\mu \frac{\partial \bar{u}_{i}}{\partial x_{j}}\right)-\frac{\partial \tau_{i j}}{\partial x_{j}}
\end{aligned}
$$

where the over-bars denote filtered quantities. The subgrid scale (SGS) stress $\tau_{i j}$ as the extra term in Eq. (11), which must be modeled, is defined as follows:

$\tau_{i j}=\rho\left(\overline{u_{i} u_{j}}-\overline{u_{i} u_{j}}\right)$

The Boussinesq hypothesis is employed, in which the SGS stress is computed from

$\tau_{i j}-\frac{1}{3} \tau_{k k} \delta_{i j}=-2 \mu_{t} \bar{S}_{i j}$

where $\mu_{t}$ is the SGS turbulent viscosity. Isotropic part $\tau_{k k}$ is not modeled but added to the filtered static pressure term. $\bar{S}_{i j}$ is the rate-of-strain tensor for the resolved scale defined by

$\bar{S}_{i j} \equiv \frac{1}{2}\left(\frac{\partial \bar{u}_{i}}{\partial x_{j}}+\frac{\partial \bar{u}_{j}}{\partial x_{i}}\right)$

The SGS turbulent viscosity $\mu_{t}$ is closed by the SmagorinskyLilly model, and modeled by $\mu_{t}=\rho L_{s}^{2} \sqrt{2 \bar{S}_{i j} \bar{S}_{i j}}$, where $L_{s}$ is the mixing length for subgrid scales. It is computed by $L_{s}=$ $\min \left(\kappa d, C_{S} V^{1 / 3}\right)$, where $\kappa$ is the von Karman constant, $d$ is the distance to the closet wall, $C_{S}$ is the Smagorinsky constant set as 0.1 and $V$ is the volume of the computational cell.

\subsection{Simulation setup}

Unsteady numerical simulations are performed based on the finite-volume method with a coupled scheme by using commercial computational fluid dynamics software ANSYS Fluent (ANSYS, Inc., 2013). The equations are discretized by a second-order implicit scheme in time and a bounded central differencing scheme in space. A implicit scheme is also used for time discretization of the volume fraction equation which is compatible with the cavitation model.

The pressure staggering option is selected for pressure interpolation. The modified high-resolution interface-capturing scheme used for the volume fraction, which is more robust than explicit geometric reconstruction scheme. The unsteady cavitating flow simulations are started from a uniform flow field because the 


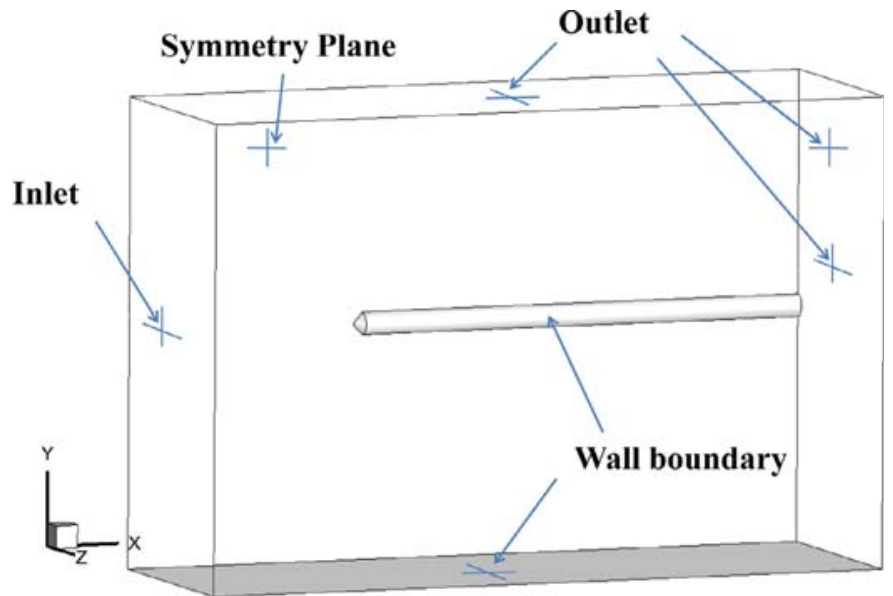

Fig. 4. The computational domain.

whole acceleration process is very short. The time step is set as $10^{-5} \mathrm{~s}$.

The computational domain is shown in Fig. 4, with half of the model considered. A semi-infinite projectile model is used, and the effect of the tail on the shoulder cavity is neglected. The model is fixed, with the free surface moving towards the model. For the velocity inlet boundary condition, the inlet velocity is set as $17.8 \mathrm{~m} / \mathrm{s}$, with no turbulent perturbations. The bottom is also set as a wall boundary. The computational domain is discretized with a blockstructured grid, which is refined around the model and near the free surface (as shown in Fig. 5). The cell number is approximately 4 million with good orthogonality.

\subsection{Verifications of numerical methods}

In order to provide some indication of how sensitive the numerical prediction, three supplementary cases are simulated, and evolutions of the cavity and free surface at typical moments are shown in Fig. 6. First, the inlet and outlet planes are extended in Case 2, and distances between the model's cone apex and boundaries are extended to twice of the original case in the $X$ direction. Second, the time step is decreased to $5 \times 10^{-6} \mathrm{~s}$ as half of the original case in Case 3. Third, the number of cells is doubled in each direction in Case 4, so the total number of cells is 8 times of the original grid and approximately 32 million.

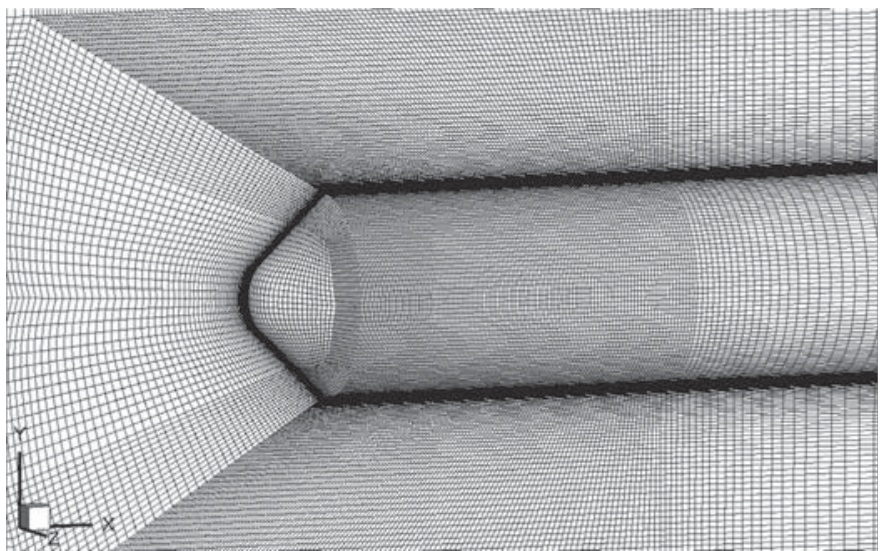

Fig. 5. Mesh near the head of the projectile.

By comparing the main features pointed by the red arrow in each figures, we can find that results agree well with each other in the aforementioned 4 cases, including: the position and inclined angle of the re-entry jet front at the moment of $4 \mathrm{~ms}$, the shape of the shedding cavity at the moment of $8 \mathrm{~ms}$, the profile of the trailing edge of the main cavity influenced by the cavity collapse at the moment of $11 \mathrm{~ms}$. In the Case 4 compared with the original case, the shedding cavity is larger in the view 4-b, and more parts remain in the view 4-c. This may be due to the slight delay of the cavity development in Case 4. Moreover, wave shapes of the free surface are very similar for all the cases at the same moment. It can demonstrate the robustness on the prediction of the transient free surface of the present method. Therefore, algorithm and grid options are appropriate in the original case, and its results are used in following contents.

\section{Results and discussion}

\subsection{Comparison of cavities evolution}

The quasi-periodic development of cavity shape and length which are obtained from the experimental and numerical results. The variation of cavity length in the first and second cycles is shown in Fig. 7, in which every cycle can be also divided into two substages. Stage 1-1 is the growth period. The re-entry jet is generated and developed firstly in stage $1-2$. The cavity sheds in stage $2-1$ and collapses in stage $2-2$, in which the re-entry jet is also

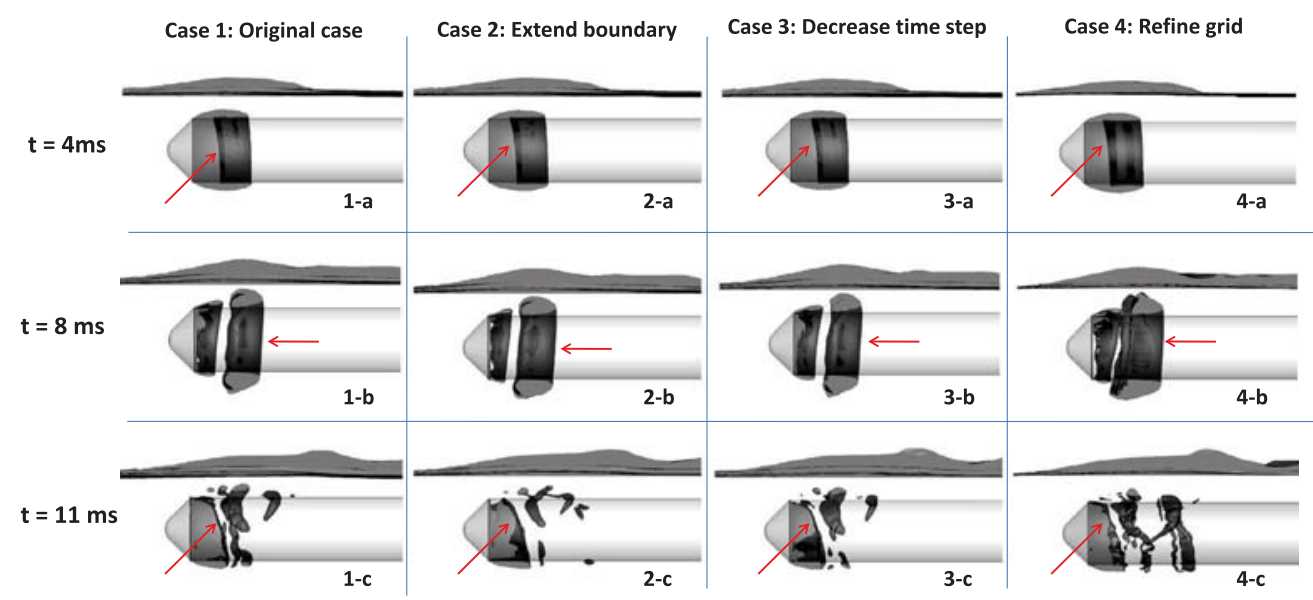

Fig. 6. Evolutions of the cavity and free surface at typical moments. Developments of the cavity and re-entry jet are at the moment of 4 ms. Cavity Shedding is at the moment of $8 \mathrm{~ms}$. Collapse of the shedding cavity is at the moment of $11 \mathrm{~ms}$. 


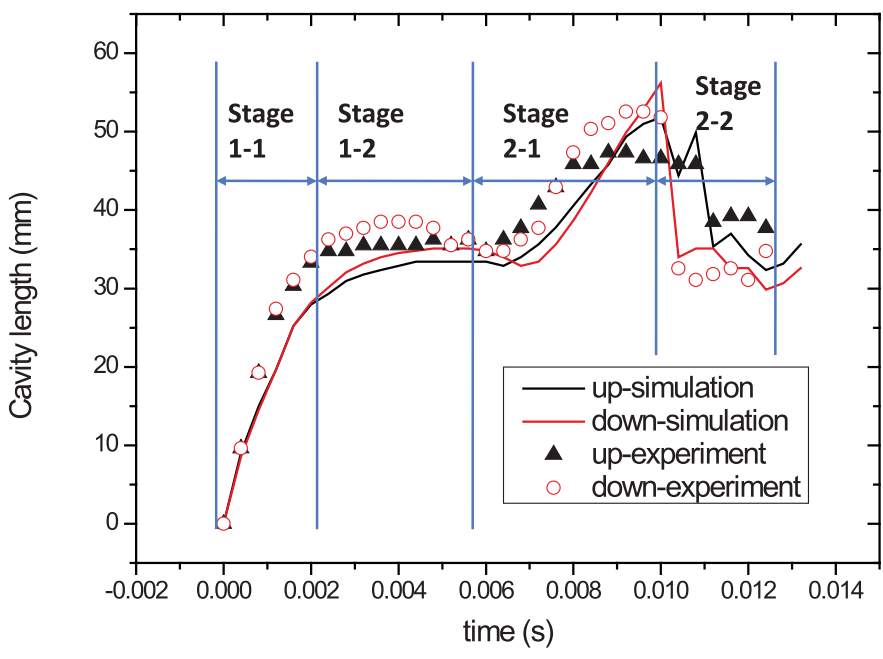

Fig. 7. The comparison of the variation of cavity length in the first and second cycles.
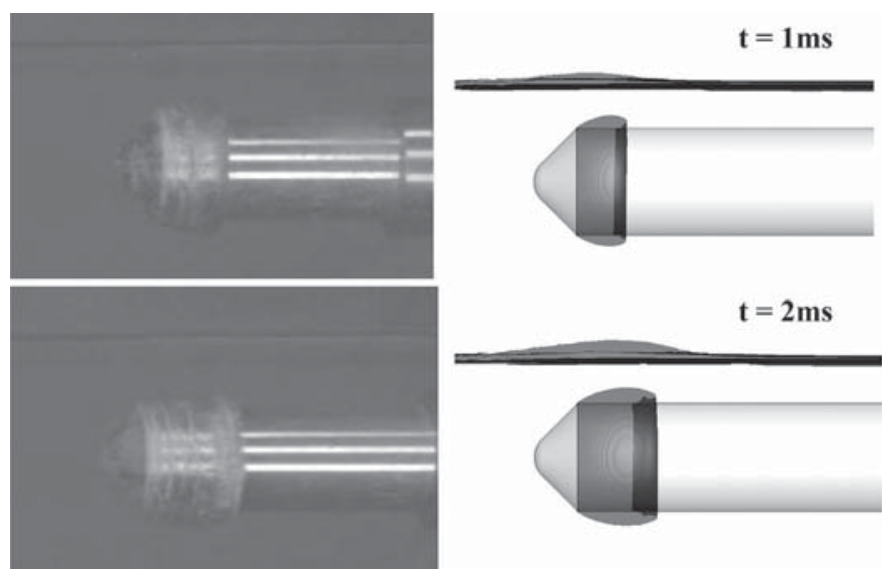

Fig. 8. Time evolution of cavitation patterns obtained from the experiment and simulation in the stage 1-1.

generated for the second time. In the last three stages, a significant difference is found between the lengths of in the upper and lower sides of the projectile. Longer cavities appear alternately.

The length development and variation of the obtained numerical results agree fairly with the experiments, including the relationship between upper and lower sides.

The characteristics of cavity development in different stages are separately analysed in the following. In stage $1-1$, which is the growth stage, the cavity is transparent and grows stably. The cavity in the lower side is slightly longer than the upper side; the difference is hardly distinguishable (as shown in Fig. 8)

In stage 1-2, when the cavity grows to a stable shape, the re-entry jet is generated and flows upstream the cavity. In the experimental observation, the re-entry jet exhibits a white foamlike flow structure. In the numerical graphs, the re-entry jet can be demonstrated clearly as a liquid-phase flow, which is close to the wall and advances to the head of the projectile. In this stage, the cavity on the upper side is shorter than the lower side, and the length is unchanged. In the following content, the result at $0.004 \mathrm{~s}$ (as shown in the middle view) is selected to investigate the mechanism of the interaction between the free surface and the cavity (as shown in Fig. 9).

Re-entry jet is one of the most important inducing factors on cavity shedding and instability. In this stage, the re-entry jet on the upper side is always in front of the lower side. The flow vectors on
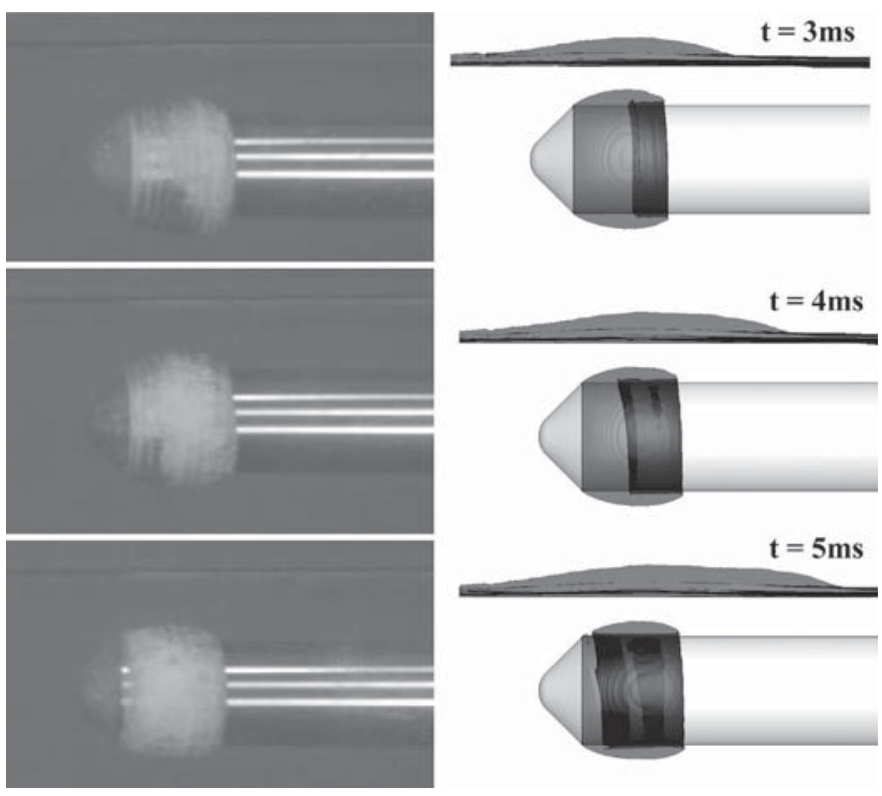

Fig. 9. Time evolution of cavitation patterns obtained from the experiment and simulation in the stage $1-2$.

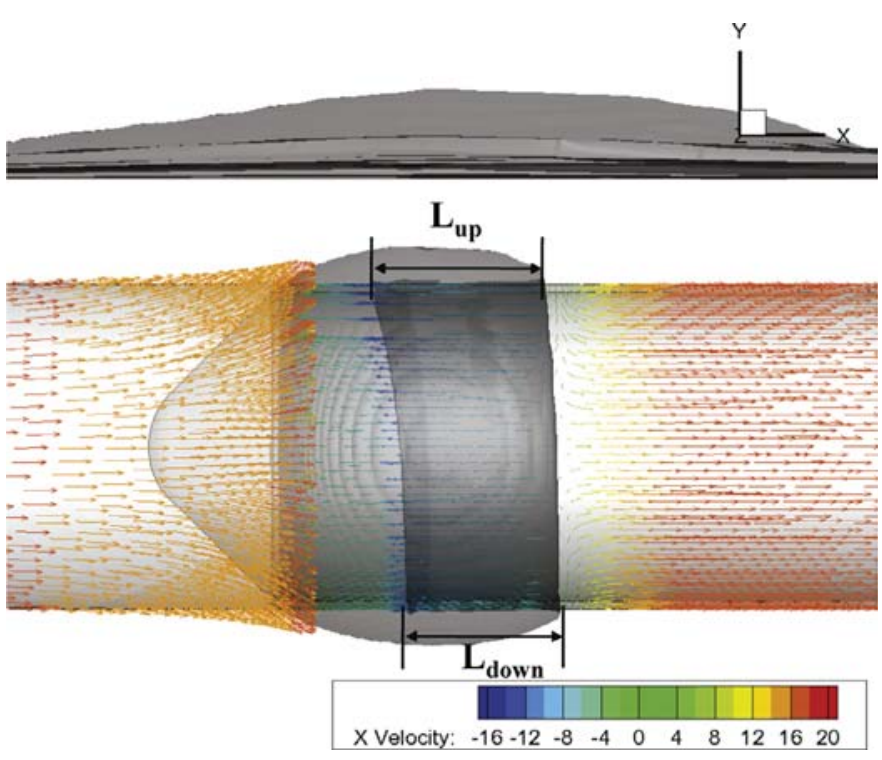

Fig. 10. The flow vectors on the surface of 1.01 diameters to show the re-entry jet

the surface of 1.01 diameters are shown in Fig. 10 at $0.001 \mathrm{~s}$. The re-entry jet is in the liquid-phase region, and the flow direction is opposite to the main flow that faces the leading edge.

A comparison of the fronts of re-entry jet between the numerical and experimental results is shown in Fig. 11. The figure shows that the re-entry jet inception is set as the original point of the time coordinate. The re-entry jet began almost simultaneously on the upper and lower sides. The velocity of the re-entry jet is higher at the beginning and slightly lower at the end. The variation scale is small, and the front moves much linearly with time. The average velocity of the re-entry jet on the upper side is slightly higher than the lower side. The front on the upper side is much closer to the leading edge of the projectile. Fair agreement is achieved between the numerical and experimental results.

When the re-entry jet arrives at the shoulder, it cuts off the cavity by interfering with the main flow. The re-entry jet on the upper side reaches earlier, thereby inducing the cavity to shed 


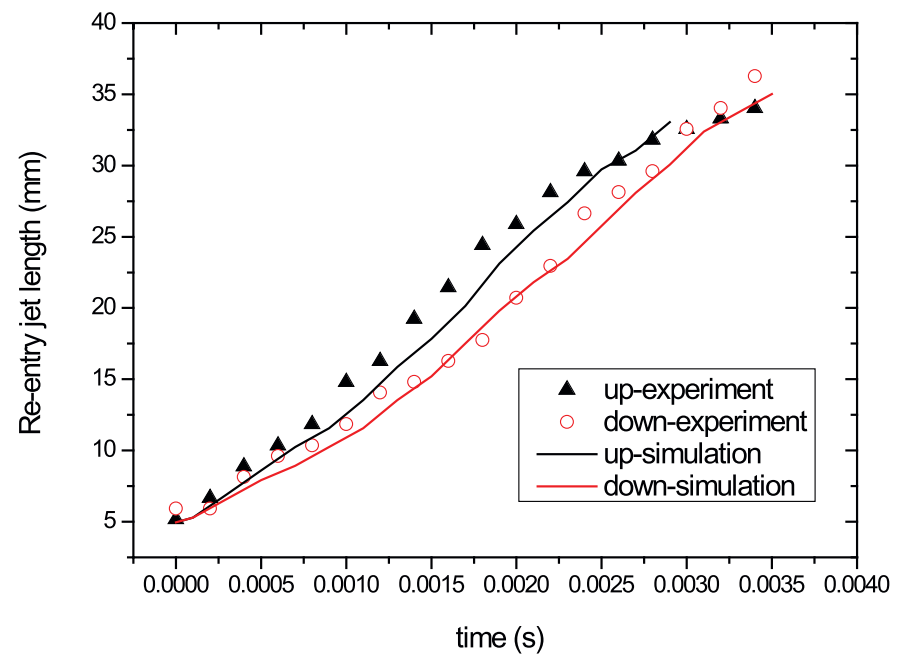

Fig. 11. The comparison of the front of re-entry jet between the numerical and experimental results in stage 1-2.
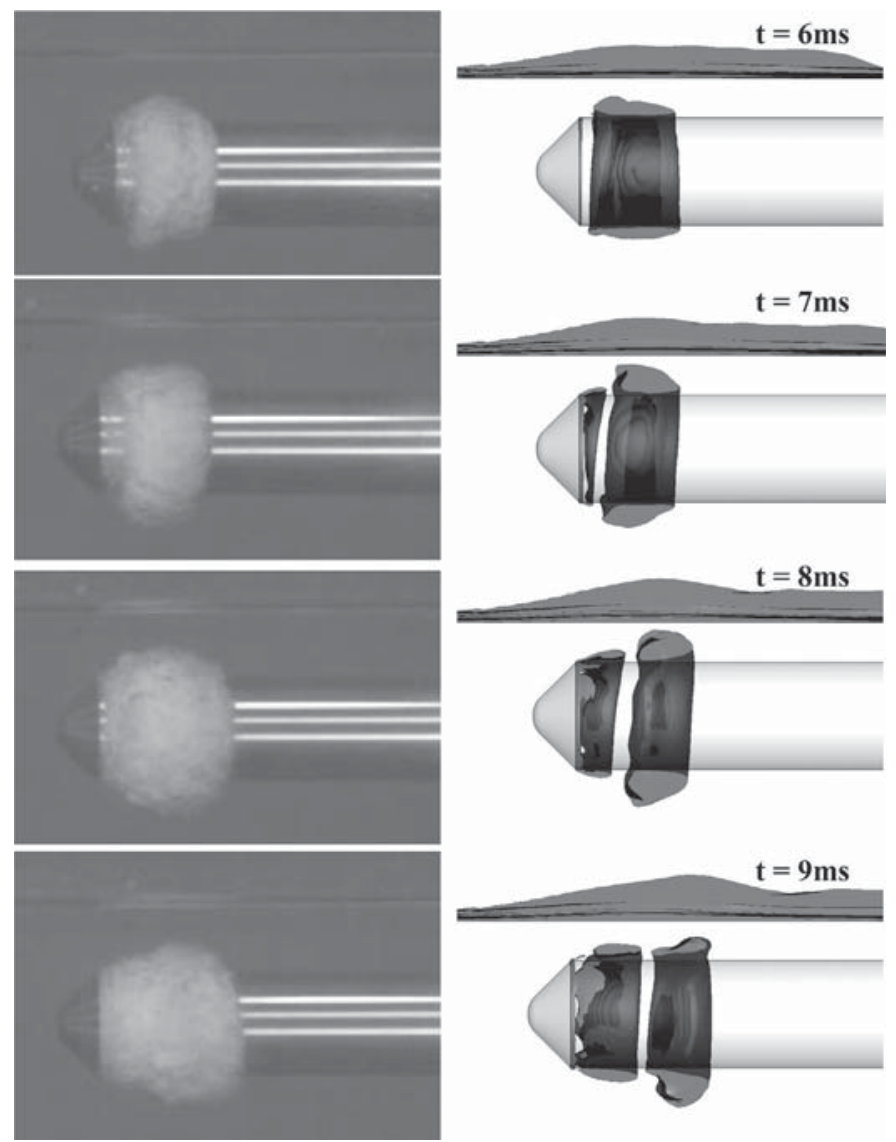

Fig. 12. Time evolution of cavitation patterns obtained from the experiment and simulation in the stage $2-1$.

before the lower side. As shown in Fig. 12, the thickness of the shedding cavity on the upper side is larger than that on the lower side. When the shedding cavity flows downstream, the cavity on the upper side becomes longer than the lower side for the first time (as shown in Fig. 12). As the cavity on the lower side sheds along with the main flow, it becomes thicker (as shown in Fig. 12). Then, when the shedding cavity is almost parallel to the newly

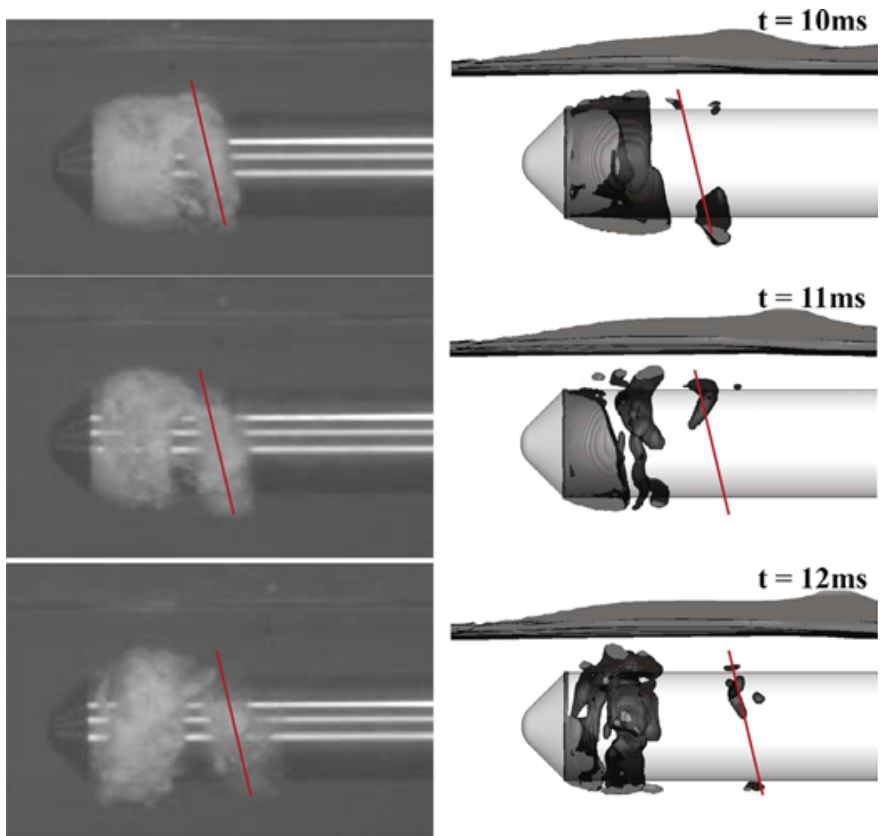

Fig. 13. Time evolution of cavitation patterns obtained from the experiment and simulation in the stage $2-2$.

generated cavity, the thickness becomes similar on both sides, and the cavity on the lower side grows longer again.

As the shedding cavity flows downstream, the strength of its concomitant vortex becomes weaker. Therefore, the shedding cavity begins to collapse at the upstream part on the lower side (a detailed investigation of the motion and collapse of the shedding cavity can be found in the reference (Yu et al., 2014) ). The profile of collapsing cavity is nonaxisymmetric and is an inclined line from the left of the upper side to the right of the lower side. The shedding cavity is closer to the newly generated cavity on the upper side, and collapse occurs as a result. If the shedding cavity is considered a whole bubble ring, then the collapse of the upper part is actually delayed by the effect of the newly generated cavity (as shown in Fig. 13).

\subsection{Effect of the free surface on the cavity shape}

Flow field values along two typical stream traces are selected for comparison to analyze the effect of the free surface(as shown in Fig. 14). One trace is above the projectile, while the other is below it. The $x$ coordinates of the start points of the traces are all located one radius away in front of the shoulder point, while the $y$ coordinates are located ten percents of the radius away from the axis, respectively. The traces are close to the wall surface, so they can be considered as the profiles of the cavities, which corresponds to the classic theory of cavitating flow.

The radical velocity distributions on the traces are compared as follows. With respect to the absolute values, the initial Y velocity on the shoulder position is higher in the upper flow field (as shown in Fig. 15). The deceleration of the upper flow is also larger, thereby inducing a high minus value at the cavity closure, which will form a high-pressure region. This distribution agrees with the theoretical results, which reflects that the mass of jet in the upper field is small.

The shapes of the stream traces are similar to the cavity profiles, as shown in Fig. 16. The cavity on the upper side is thicker and shorter, which is consistent with the numerical results obtained by Faltinsen and Semenov (2008) on the stable supercavity. 


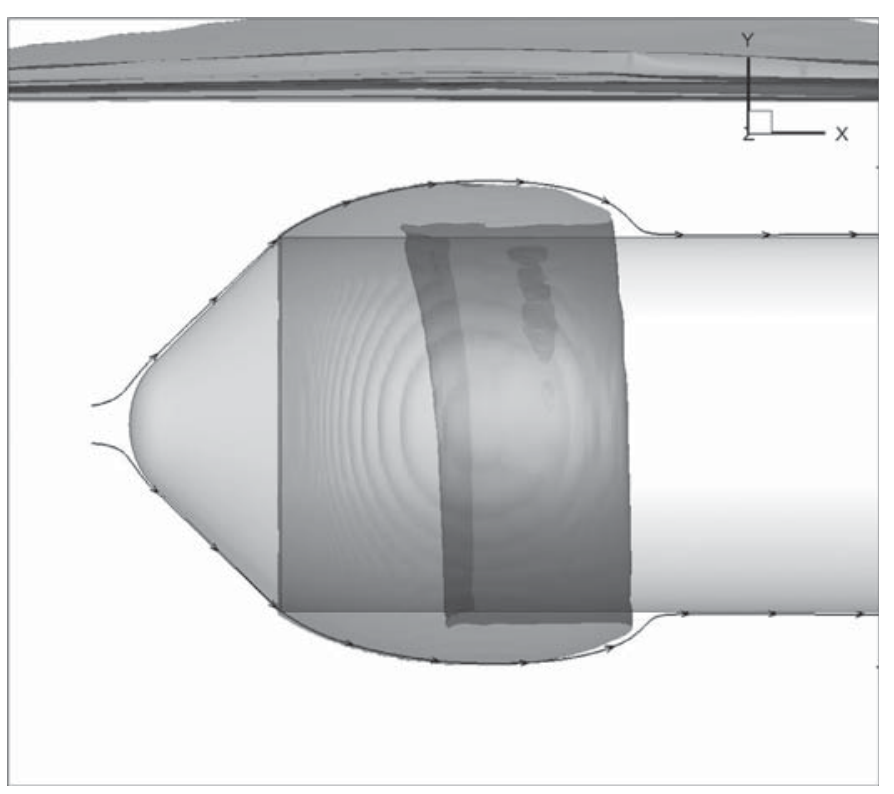

Fig. 14. Typical stream traces around the cavity (0.004 s).

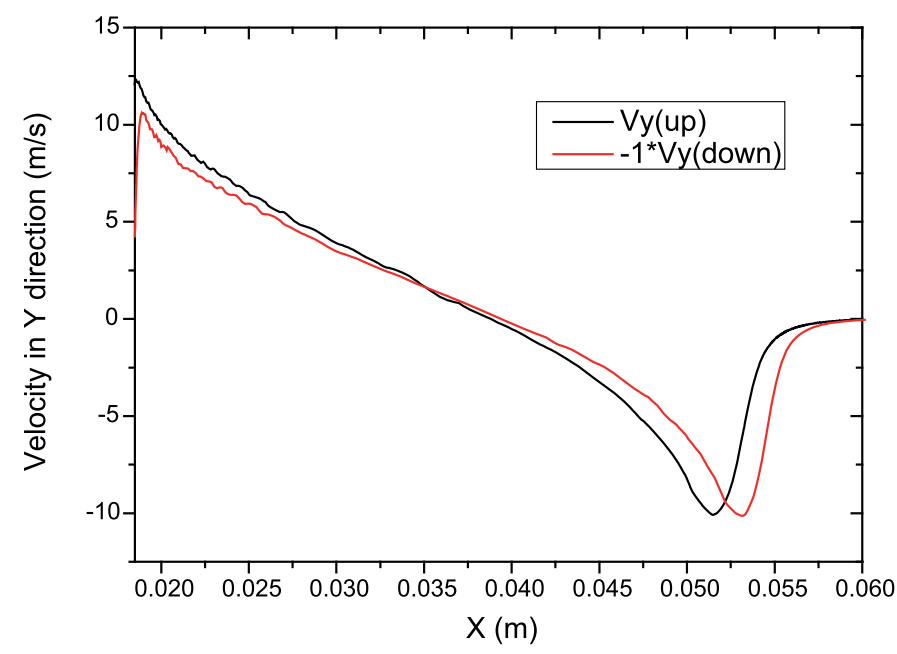

Fig. 15. Comparison of $Y$-direction velocity distributions along stream traces on the upper and lower side of the projectile in numerical results.

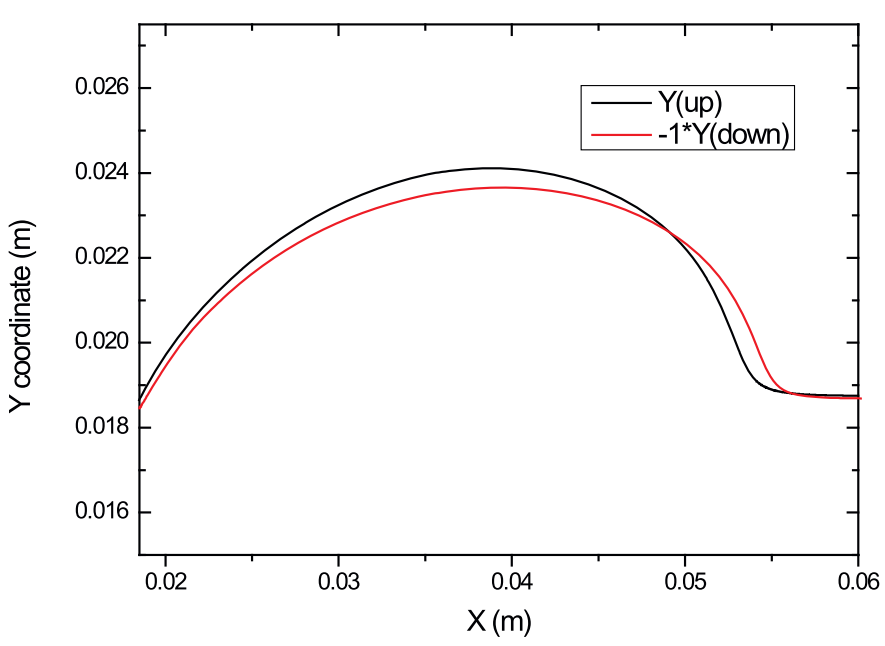

Fig. 16. Comparison of stream traces shapes(the cavity profiles) on the upper and lower side of the projectile in numerical results.
These results indicate that the constraint of the surrounding flow field becomes weak when the free surface exists. Consequently the water layer between the projectile and the free surface can easily move outwards in the radial direction and also move inwards under the pressure difference between the ambient pressure and the pressure in the cavity (as shown in Fig. 15). So the profile has larger curvature near the free surface (as shown in Fig. 16).

\subsection{Analysis on the tangential velocity evolution}

Non-axisymmetric features of cavities are mainly caused by the effect of the free surface. If the projectile is in the cylindrical coordinate, pressure distributions are non-axisymmetric either. Therefore the tangential velocity is generated by the tangential pressure gradient, which can demonstrate the relationship of flow fields in sections with various tangential coordinates.

The distributions of tangential velocity around the projectile in the stage 1-2 are as shown in Fig. 17(a)-(b). Obvious tangential velocities only exist around the shoulder as local velocity fluctuations. The tangential component is small of the re-entry jet velocity.

In the shedding stage $2-1$, the re-entry jet arrives at the shoulder point and cut off the remaining cavity, inducing new cavities. Upward tangential components exist in the new-generated cavities in the whole shedding stage. Corresponding fluid contains much liquid water phase, which make the cavity on the upper side detach from the wall and upturn more easily (as shown in Fig. 17 (c)(f)).

In the collapse stage $2-2$, high pressure pulses are generated by the cavity collapse at the cavity closure. Because of the nonaxisymmetric characteristics of shedding cavity and collapse sequences, the tangential distribution of high pressure regions is significantly uniform, which also generates many local vortexes in the cylinderical surface. Consequently the lateral and vertical forces are also much larger than those in the former stages (as shown in Fig. 17 (g)-(h)).

\subsection{Effect on shedding vortex structures}

The motion of shedding cavitation clouds has strong correlation with the vortex motion. The flow structures can be visualized based on the Q-criterion. In the present study, the iso-surface of $Q=50,000$ is shown to represent the vortex core, and the color represents the velocity component in the axial direction. Vortex structures in the shedding process are as shown in Fig. 18. The shedding process around the projectile is not synchronized, and the shedding vortex is inclined. The broken vortex is thinner on the upper side under the effect of the free surface.

\subsection{Effect of cavity on the wave elevation}

The wave and cavity profiles on the upper side of the projectile at $0.004 \mathrm{~s}$ are shown in Fig. 19. The maximum height of the wave in the cavitation case is higher than that of the wave in the non-cavitation case, but the difference is about half of the cavity thickness. The free surface can still provide constraint effects to the flow and cavity because of the wave making resistance. The actual constraint effect is between the effects of the infinite water field and the fully free condition.

\section{Conclusions}

An experiment on the cloud cavitating flow around the axisymmetric projectile near the free surface has been presented in this paper. A numerical simulation is performed based on VOF and LES methods. Unsteady behavior of the cloud cavity under the effect 


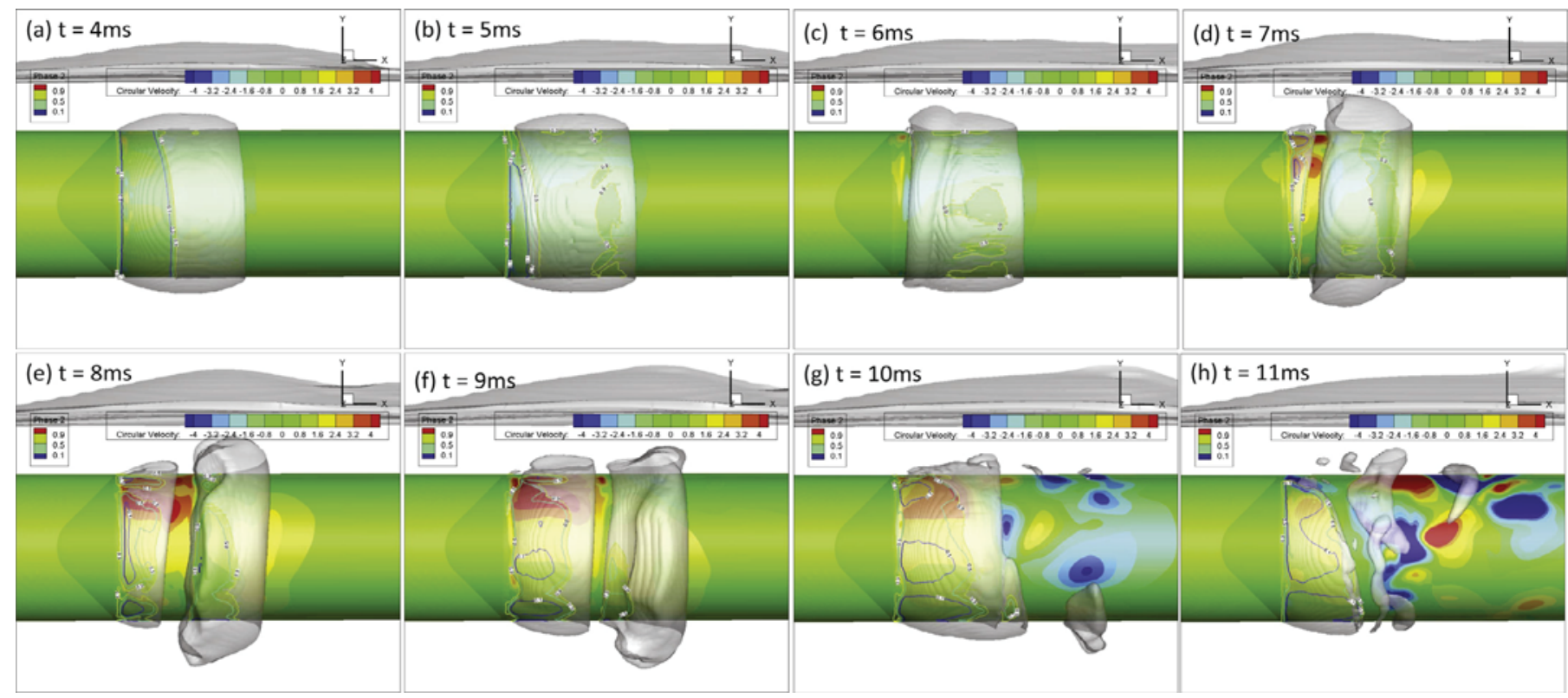

Fig. 17. Contours of tangential velocity on a cylindrical surface (The surface is set $5 \%$ of the diameter away from the projectile wall. The positive flood value means that the flow is upwards in the region where $Z$ coordinate is positive in the cartesian coordinate. The line represent the volume fraction of liquid water phase).

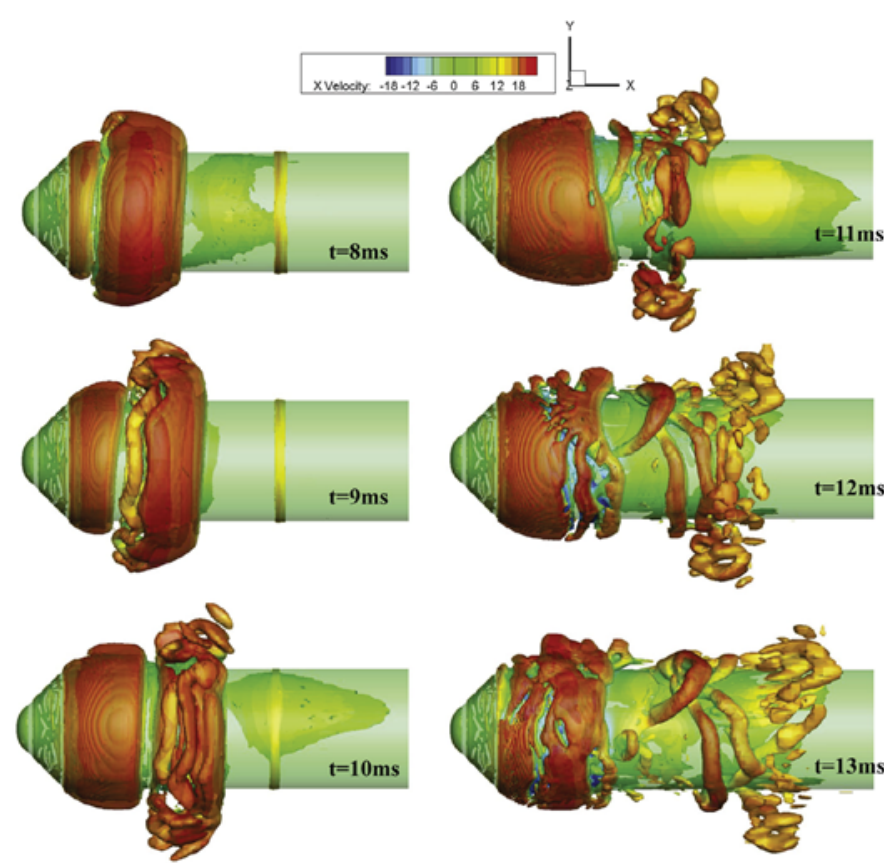

Fig. 18. Vortex structures in the cavity shedding process $(Q=50,000$, and the color represents the velocity component in the axial direction).

of the free surface is obtained, and good agreements are achieved between the numerical and experimental results.

The cavity evolution on the upper side of projectile is remarkably different compared to the lower side under the free-surface effect. For instance, on the upper side, cavity growth is slower, the velocity of the re-entry jet is higher, the cavity sheds in advance, and the position of shedding cavity collapse is closer to the main cavity.

Results indicate that because the flow stream around the upper surface is thin, changing its direction under the effect of pressure difference inside and outside the cavity is easy. The constraint

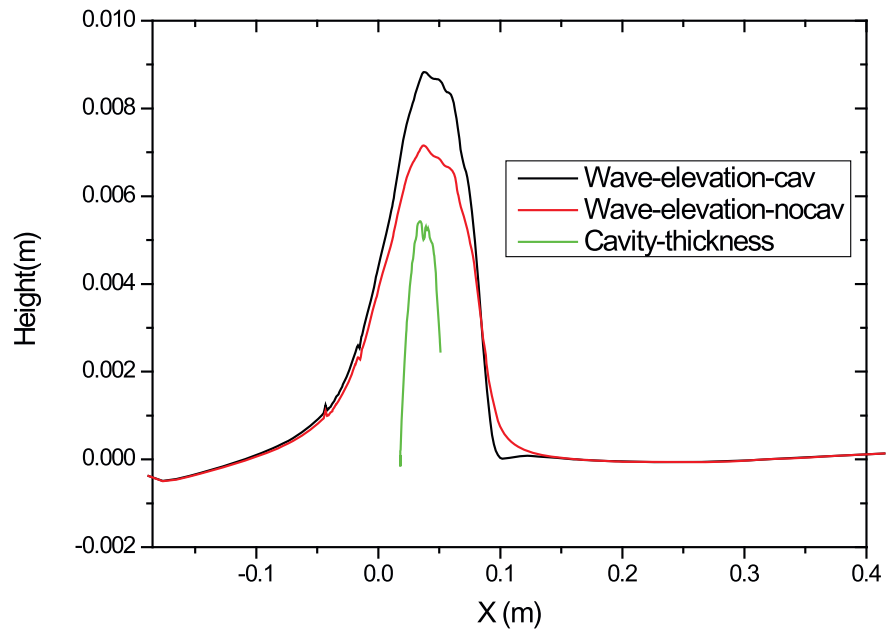

Fig. 19. Wave profiles with and without cavitation and the cavity profile on the upper side.

variation caused by the existence of the free surface also leads to changes in cavity length, thickness and subsequent shape evolutions.

Non-axisymmetric collapse features generate a mass of strong vortexes in the tangential surface, and the non-uniform distribution of high pressure region is also one of the most important factors to induce lateral and vertical forces on the projectile.

The heights of wave elevation in cases with and without cavitation are compared. The presence of cavitation leads to the increase in wave height, however the increment is about half of the cavity thickness. The actual constraint effect is between the effects of the infinite water field and the fully free condition.

The effect of the free surface on unsteady cloud cavitation is a complicated issue. With the variation of distance, the effect may show different situations of quantitative and qualitative changes. This paper presents only the results of a body of typical shape and under a typical working condition, and more in-depth analysis should be conducted in the future. 


\section{Acknowledgment}

The authors are grateful to the National Natural Science Foundation of China through grant numbers 11202215 \& 11332011. This project was also supported by the Youth Innovation Promotion Association CAS (2015015).

\section{References}

Amromin, E., 2007. Analysis of body supercavitation in shallow water. Ocean Eng. $34,1602-1606$.

ANSYS, Inc., 2013. ANSYS Fluent Theory Guide.

Bal, S., 2007. High-speed submerged and surface piercing cavitating hydrofoils including tandem case. Ocean Eng. 34, 1935-1946.

Bal, S., 2011. The effect of finite depth on 2D and 3D cavitating hydrofoils. J. Mar Sci. Tech. Jpn. 16, 129-142.

Bal, S., Kinnas, S.A., 2002. A BEM for the prediction of free surface effects on cavitating hydrofoils. Comput. Mech. 28, 260-274.

Bensow, R.E., Bark, G., 2010. Implicit LES predictions of the cavitating flow on a propeller. ASME J. Fluids Eng. 132, 041302.

Chen, X., Lu, C., Li, J., Chen, Y., 2011. Properties of natural cavitation flows around a 2-d wedge in shallow water. J. Hydrodyn. 23, 730-736.

Coutier-Delgosha, O., Deniset, F.O., Astolfi, J.A., Leroux, J.B., 2007. Numerical prediction of cavitating flow on a two-dimensional symmetrical hydrofoil and comparison to experiments. ASME J. Fluids Eng. 129, 279-292.

Coutier-Delgosha, O., Fortes-Patella, R., Reboud, J.L., 2003. Evaluation of the turbulence model influence on the numerical simulations of unsteady cavitation. ASME J. Fluids Eng. 125, 38-45

Coutier-Delgosha, O., Reboud, J.L., Delannoy, Y., 2003. Numerical simulation of the unsteady behaviour of cavitating flows. Int. J. Numer. Methods Fluids 42, 527-548.

Coutier-Delgosha, O., Stutz, B., Vabre, A., Legoupil, S., 2007. Analysis of cavitating flow structure by experimental and numerical investigations.. J. Fluid Mech 579 $\underline{171-222 .}$

Dawson, T.E., 1959. An experimental investigation of a fully cavitating two-dimensional flat plate hydrofoil near a free surface.. California Institute of Technology Ph.D. thesis.

Dittakavi, N., Chunekar, A., Frankel, S., 2010. Large eddy simulation of turbulent-cavitation interactions in a venturi nozzle. ASME J. Fluids Eng. 132, 121301.

Faltinsen, O.M., 2005. Hydrodynamics of High-speed Marine Vehicles. Cambridge University Press..

Faltinsen, O.M., Semenov, Y.A., 2008. The effect of gravity and cavitation on a hydrofoil near the free surface. J. Fluid Mech. 597, 371-394.

Franc, J.-P., Michel, J.-M., 2005. Fundamentals of Cavitation. Springer Science \& Business Media.

Gnanaskandan, A., Mahesh, K., 2015. A numerical method to simulate turbulent cavitating flows. Int. J. Multiph. Flow 70, 22-34.

Hu, C., Wang, G., Chen, G., Huang, B., 2014. A modified PANS model for computations of unsteady turbulence cavitating flows. Sci. China Phys. Mech. 57, 1967-1976.
Huang, B., Wang, G., 2011. Partially averaged Navier-Stokes method for time-dependent turbulent cavitating flows. J. Hydrodyn. 23, 26-33.

Huang, B., Zhao, Y., Wang, G., 2014. Large eddy simulation of turbulent vortex-cavitation interactions in transient sheet/cloud cavitating flows. Comput. Fluids 92 113-124.

Ji, B., Luo, X., Arndt, R.E.A., Wu, Y., 2014. Numerical simulation of three dimensiona cavitation shedding dynamics with special emphasis on cavitation-vortex interaction. Ocean Eng. 87, 64-77.

Ji, B., Luo, X., Wu, Y., Peng, X., Xu, H., 2013. Partially-averaged Navier-Stokes method with modified k-epsilon model for cavitating flow around a marine propeller in a non-uniform wake. Int. J. Heat Mass Transf. 55, 6582-6588.

Ii, B., Luo, X.W., Arndt, R.E.A., Peng, X., Wu, Y., 2015. Large eddy simulation and theoretical investigations of the transient cavitating vortical flow structure around a NACA66 hydrofoil. Int. J. Multiph. Flow 68, 121-134.

Karim, M.M., Prasad, B., Rahman, N., 2014. Numerical simulation of free surface water wave for the flow around NACA 0015 hydrofoil using the volume of fluid (VOF) method. Ocean Eng. 78, 89-94.

Kunz, R.F., Boger, D.A., Stinebring, D.R., 1998. A preconditioned Navier-Stokes method for two-phase flows with application to cavitation. Comput. Fluids 29 , 849-875.

Liang, H., Sun, L., Zong, Z., Zhou, L., Zou, L., 2013. Analytical modelling for a three-dimensional hydrofoil with winglets operating beneath a free surface. Appl. Math Model. 37, 2679-2701

Merkle, C.L., Feng, J., Buelow, P.E.O., 1998. Computational modeling of the dynamics of sheet cavitation. In: Proceedings of the 3rd International Symposium on Cavitation. Grenoble, France.

Roohi, B., Zahiri, A.P., Passandideh-Fard, M., 2013. Numerical simulation of cavitation around a two-dimensional hydrofoil using VOF method and LES turbulence model. Appl. Math. Model. 37, 6469-6488.

Singhal, A.K., Athavale, M.M., Li, H., Jiang, Y., 2002. Mathematical basis and validation of the full cavitation model. ASME J. Fluids Eng. 124, 617-624.

Stutz, B., Legoupil, S., 2003. X-ray measurements within unsteady cavitation. Exp Fluids 35, 130-138.

Stutz, B., Reboud, J.L., 1997. Experiments on unsteady cavitation. Exp. Fluids 22, 191-198.

Stutz, B., Reboud, J.L., 2000. Measurements within unsteady cavitation. Exp. Fluids 29, 545-552.

Wang, G., Ostoja-Starzewski, M., 2007. Large eddy simulation of a sheet/cloud cavitation on a NACA0015 hydrofoil. Appl. Math. Model. 31, 417-447.

Wei, Y., Wang, Y., Fang, X., Huang, C., Duan, Z., 2011. A scaled underwater launch system accomplished by stress wave propagation technique. Chin. Phys. Lett. 28 024601.

Wu, J., Wang, G., Shyy, W., 2005. Time-dependent turbulent cavitating flow computations with interfacial transport and filter-based models. Int. J. Numer. Methods Fluids 49, 739-761.

Xie, N., Vassalos, D., 2007. Performance analysis of 3d hydrofoil under free surface. Ocean Eng. 34, 1257-1264.

Yu, X., Huang, C., Du, T., Liao, L., Wu, X., Zheng, Z., Wang, Y., 2014. Study of characteristics of cloud cavity around axisymmetric projectile by large eddy simulation. ASME J. Fluids Eng. 136, 051303.

Zhou, L., Wang, Z., 2008. Numerical simulation of cavitation around a hydrofoil and evaluation of a RNG $\kappa-\varepsilon$ model. ASME J. Fluids Eng. 130, 011302.

Zwart, P.J., Gerber, G.A., Belarmri, T., 2004. A two-phase flow model for predicting cavitation dynamics. In: Proceedings of the Fifth International Conference on Multiphase Flow. Yokohama, Japan. 